\title{
Assisting Difficult Liver Operations Using 3D Printed Models
}

\author{
Andreas Tooulias ${ }^{1}$, Georgios Tsoulfas ${ }^{2}{ }^{\oplus}$, Vasileios Papadopoulos ${ }^{1} \oplus$, Maria Alexiou ${ }^{1}$, Ion-Anastasios $\operatorname{Karolos}^{3}{ }^{\circledR}$, \\ Christos Pikridas $^{3}(\mathbb{1})$ and Vassilios Tsioukas ${ }^{3, *}$ \\ 1 1st Surgical Department, School of Medicine, Aristotle University of Thessaloniki, 56403 Thessaloniki, Greece; \\ astooulias@gmail.com (A.T.); papadvas@auth.gr (V.P.); mvalexiou@gmail.com (M.A.) \\ 2 Department of Transplantation Surgery, School of Medicine, Aristotle University of Thessaloniki, \\ 54642 Thessaloniki, Greece; tsoulfasg@auth.gr \\ 3 Department of Geodesy and Surveying, School of Rural and Surveying Engineering, Aristotle University of \\ Thessaloniki, 54124 Thessaloniki, Greece; ikarolos@auth.gr (I.-A.K.); cpik@auth.gr (C.P.) \\ * Correspondence: tsioukas@auth.gr
}

Citation: Tooulias, A.; Tsoulfas, G.; Papadopoulos, V.; Alexiou, M.;

Karolos, I.-A.; Pikridas, C.; Tsioukas, V. Assisting Difficult Liver Operations Using 3D Printed Models. Livers 2021, 1, 138-146. https://doi.org/10.3390/ livers1030013

Academic Editor: John N. Plevris

Received: 1 August 2021

Accepted: 30 August 2021

Published: 1 September 2021

Publisher's Note: MDPI stays neutral with regard to jurisdictional claims in published maps and institutional affiliations.

\begin{abstract}
Liver cancer is estimated to be the fifth most common in the world, while it is also considered the third leading cause of cancer death. In cases of primary liver cancer, surgery in combination with chemotherapy and radiotherapy can lead to a complete cure or significantly increase the patient's life expectancy. Since the liver is an organ that performs several critical functions in the human body, the precise estimation of the disease (position and size of tumors and its vicinity to vessels) plays a vital role in a successful operation. In some cases, the removal of the tumor may be successful, but the percentage of the hepatic remnant may not be sufficient to sustain life. Therefore, accurate imaging of the tumor of the liver and proper planning of a difficult surgery to remove tumor(s) from a patient's liver can be a lifesaver and lead to a complete cure of the disease. The aim of the present study is the initial accurate representation of the liver (parenchyma, tumors, vessels) as a digital three-dimensional (3D) model using advanced image processing and machine learning techniques and its 3D printing in 1:1 scale representing the full size of the liver with the tumor(s). A model of this type has been used at our University surgical department to plan complex hepatobiliary surgeries, provide more accurate information to the patients and their families, as well as improve the training of medical students and resident surgeons and fellows.
\end{abstract}

Keywords: liver cancer; 3D printing; 3D printing technology; 3D modeling; computerized tomography scans; hepatectomy; surgical planning; surgical imaging; surgical navigation

\section{Introduction}

Three-dimensional (3D) printing has received a lot of publicity recently. The reasons are many, but perhaps the main one is the reduction of the cost of both consumables and the 3D printing devices, as well as the wide availability of specialized open-source software that are necessary for the production of 3D printing [1]. However, its application in medical science may yet be more limited than one would expect, mainly due to the strict rules that one must follow in order to apply an innovative medical treatment.

In the last few years, the global scientific society has focused its attention on 3D modeling and 3D printing technology. This relatively new technology has a broad range of applications [2-5], with the medical industry not to be excluded from this evolution. This is due to the fact that $3 \mathrm{D}$ printers are faster, cheaper, and easier to use when compared with similar technologies, parameters that make 3D printing a cost-effective and time-saving method.

3D printers are not an invention of recent years, since the first successful application of the prosthetic process to create physical models from digital copies was made in the mid-1980s by Charles, W. Hull [6]. Nowadays, there is a wide variety of 3D printers that differ in the technology used to produce the models, materials, and costs. Like traditional 
printers, 3D printers use a variety of technologies to create their final products. More information about the types and techniques used for 3D printing can be found in relevant publications in the international literature [7].

Concerning the different medical specialties and subspecialties [8,9], there have been several important and remarkable efforts made during the last decade for this technology to constitute a valuable tool. Education and training on human organ models, profound study of disease models, education and training of students and residents, preoperative planning, intraoperative assessment of complex surgical operations that demand accuracy, postoperative evaluation, and patient counseling are fields where 3D printing is involved [10]. It appears to be a valuable tool for the surgeon in order to plan complex surgical operations carefully, with great accuracy and with fewer complications.

The current study is part of research funded by Greek National and European funds for 3D liver model printing and imaging modality development.

\section{Materials and Methods}

The use of X-ray examinations has led to a revolution in medical science and especially in the surgical specialties as the surgical team is able to investigate the inner body structure, comprehend in detail an organ's anatomy and localize the underlying damage before the surgery. From the beginning, plain radiography had as its main scope the mapping and visualization of the anatomy of the patient's tissues in order to assist in a demanding surgery. However, plain radiography could not provide the 3D information of the imaged body structure.

The development of tomographic systems of computerized tomography (CT) and magnetic resonance imaging (MRI) gave even more value and functionality to medical imaging. The series of tomography images provide important 3D information (shape, size, structure) of the inner body organs and their damages, but the visualization of the 3D anatomy is not easy as the demonstration of a solid 3D model is made on flat two dimensional (2D) screens (in general imaging peripherals) and the perception of the third dimension is subject to the physician's experience since it is done through $2.5 \mathrm{D}$ technologies [11]. These 2D graphical projections and similar techniques are used to produce images or scenes that simulate the appearance of $3 \mathrm{D}$ objects.

The standard deliverables of a CT or MRI examination is always a series of DICOM (digital imaging and communications in medicine) images characterized by high geometric accuracy and high radiometric resolution (12-bit information on grayscale values), rendering the material density of a tissue during the recording of each examination section. The intense (bright) pixels represent bones, while the darker ones, depending on their physical properties, represent soft molecules (muscles, fat, visceral organs, etc.). The intensities in the scale of gray values on the DICOM images are related to the tissues' densities. More specifically, trapped air inside the stomach or any other organ appears in darker grayscale values, while bones and blood enriched with shading fluid traveling inside the vessels appear in lighter grayscale values [12].

Although the information recorded is 3D, what can be directly and almost automatically extracted as a 3D model is the whole part of the human body that is scanned by the tomographic instrument. Isolation of specific tissue sections recorded by CT scans should be based on the density (variations in the grayscale) of the pixels belonging to specific tissues, e.g., the liver. In general, due to the proximity and indistinguishable boundaries between the internal human organs, the separation of the tissues belonging to specific organs is unclear (Figure 1). 


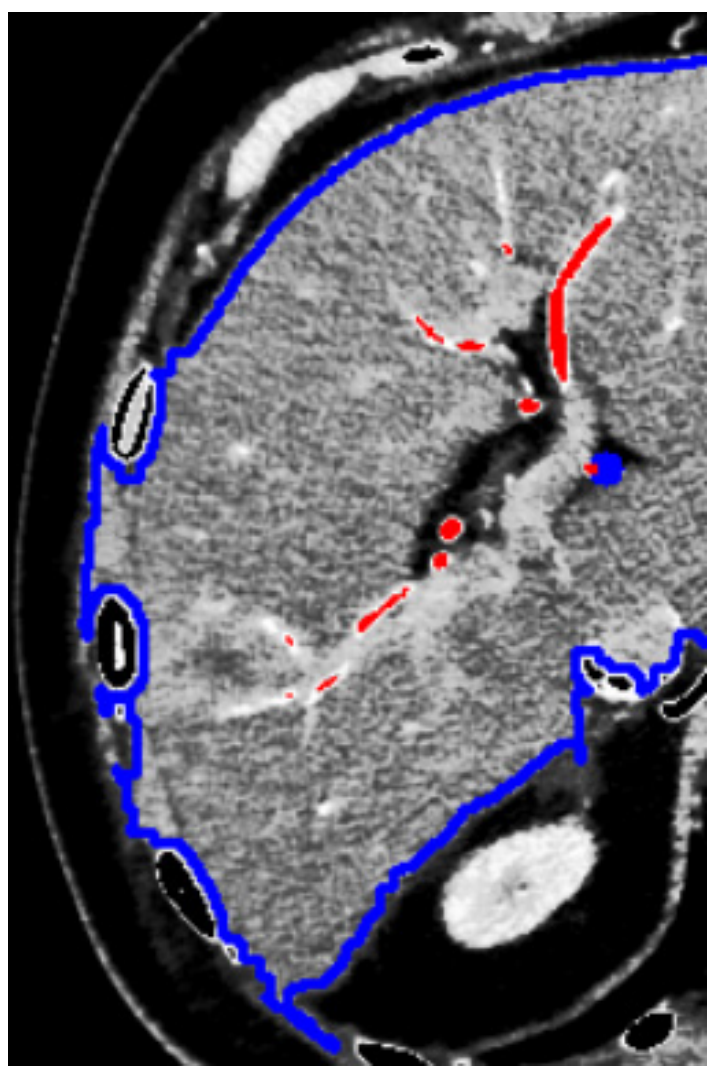

Figure 1. Problems in liver segmentation. The similar density of the hepatic parenchyma and adjacent neighboring tissues is a significant problem in separating the liver and therefore creating the complete 3D model. The blue color line is providing the border of the liver as it has been defined by a simple digital image processing algorithm, while red areas inside the liver are depicting the vessels which are visible at the specific image/slice of the DICOM image series.

The application of simple digital image processing techniques (e.g., threshold filters, region growing, and other segmentation techniques) certainly helps to isolate the tissues belonging to a specific visceral organ, especially in the case of the liver. In particular, the use of a three-phase CT scan in high resolution (providing a distance of less than $1 \mathrm{~mm}$ in successive sections) allows the separation of different structures within the hepatic parenchyma (vessels and organ pathology).

The first approach to creating a 3D model of a patient's liver involves the processing of three-phase computed tomography [13]. The present work describes the efforts made in the last two years to create models of internal human organs from synthetic materials through $3 \mathrm{D}$ printing to assist in difficult surgeries, and in particular, for the resection of liver tumors. The overall effort began with the submission of a proposal for funding to the Greek Research and Technology Agency in June 2017 and continues in the framework of the implementation of the Research Program with the acronym Liver3D co-financed by the European Union and Greek national funds through the Operational Program Competitiveness, Entrepreneurship and Innovation, under the call RESEARCH-CREATE-INNOVATE (project code: T1EDK03599) and aims at the cooperation of research institutes with Small or Medium Enterprises (SMEs) for the transfer of know-how and to encourage innovation.

Our first approach was based on creating a 3D model and inner structure of a patient's liver. This involved the processing of three-phase CT scan in three stages:

Stage (1) Extraction of the hepatic parenchyma by fully automated or semi-automated methods by delineation of the liver boundaries from neighboring organs in each image of the arterial phase (Figure 2). 


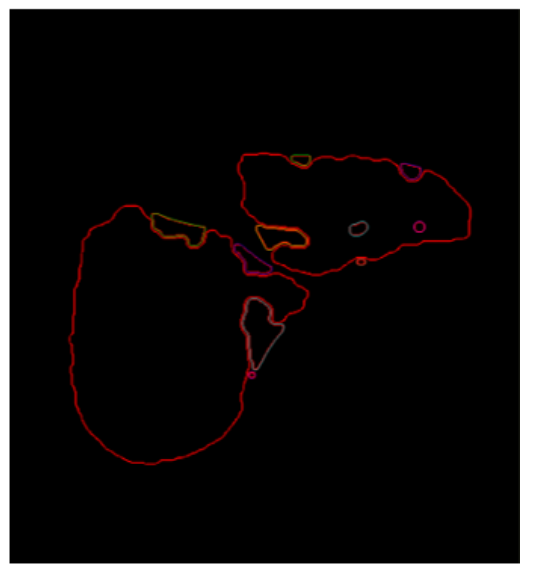

(a)

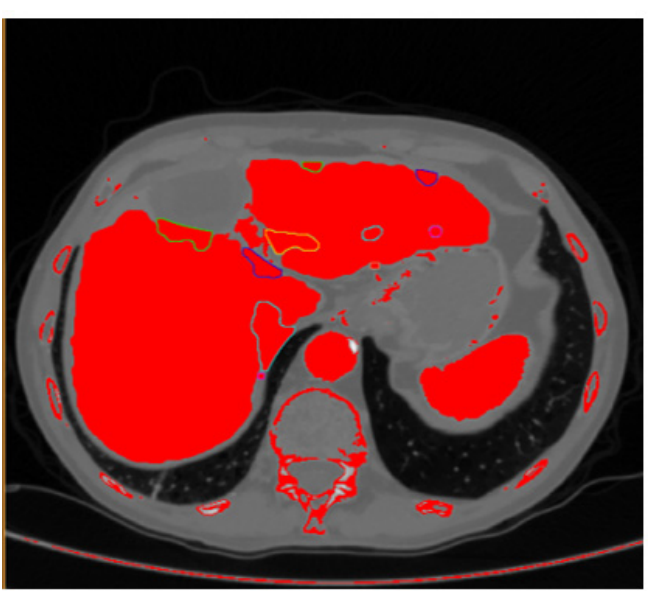

(b)

Figure 2. Liver segment extraction. Extraction of hepatic parenchyma (a) by fully automated or semi-automated methods by delineating liver boundaries from neighboring tissue (b). The procedure was applied in each one of the DICOM images of the artery CT scan phase.

Stage (2) Extraction of the lesion and vessels within the limits of the parenchyma (already limited in every CT scan image from the previous processing stage).

Stage (3) Extraction of the points belonging to the surfaces of the parenchyma, blood vessels, and the lesion and creation of models using model reconstruction algorithms (Figure 3).

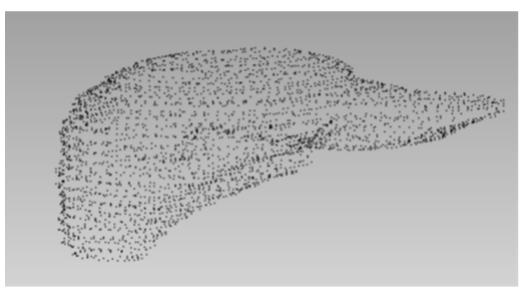

(a)

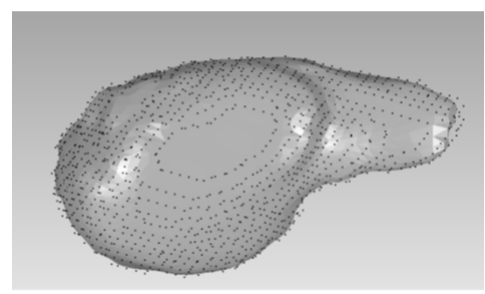

(b)

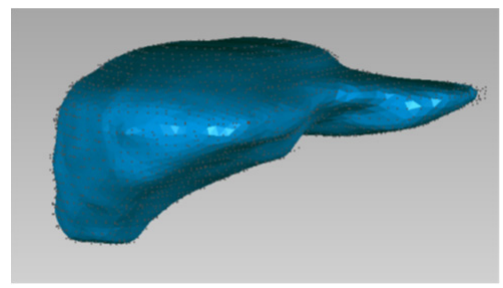

(c)

Figure 3. Point cloud and surface models of a patients' liver. Points extracted on the outer surface of the liver segment in every DICOM image of a CT scan generated the complete point cloud (a) leading to the top view (b) and front view (c) presentation of the parenchyma.

Although the application of automatic segmentation gave acceptable results, the process of extracting the complete model of the liver parenchyma was not sufficiently automated. For this reason, artificial intelligence and deep learning techniques were used to extract the models of both the parenchyma but also of the organ pathology (i.e., tumors, hemangioma, etc.) [14]. In the first case, where this algorithm was applied, only the model of the parenchyma and the tumor were extracted and printed independently with different colors (Figure 4) and on a 1:1 scale.

The specific printed model (Figure 4) was used as a guide for the therapeutic approach a few days before the surgery (Figure 5), as well as for informing the patient. The research protocol and the study were approved by the Investigational Review Board (IRB) of the hospital where the research was conducted. Informed consent was obtained from all subjects involved in the study. 


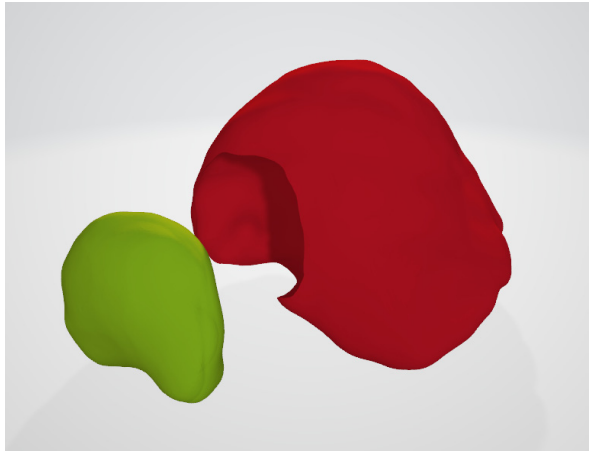

(a)

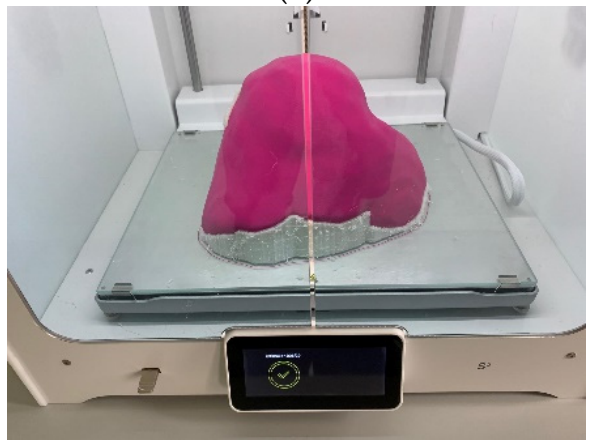

(c)

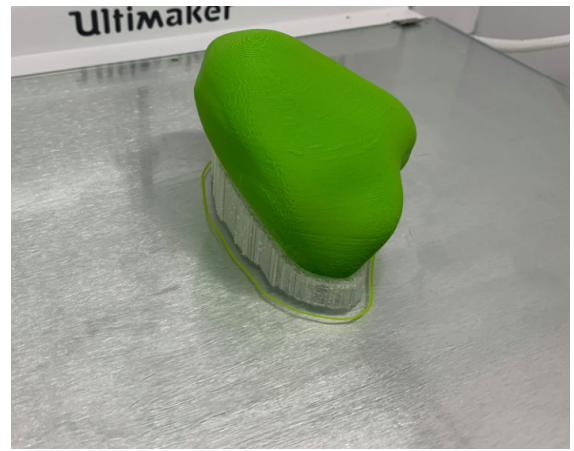

(b)

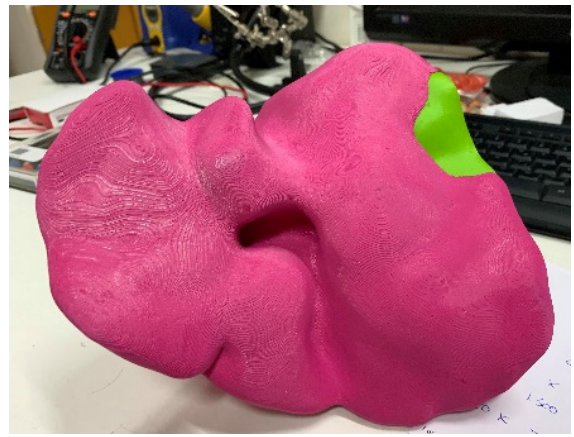

(d)

Figure 4. Digital and printed models of a patient's liver. (a) The digital parenchyma (in red color) and tumor (in green color) volume models were created using special procedure and software. The printing of the two 3D models, tumor in green color (c) and parenchyma (b) in red color, is demonstrated on the 3D printer table. The models were later integrated on a single object (d).

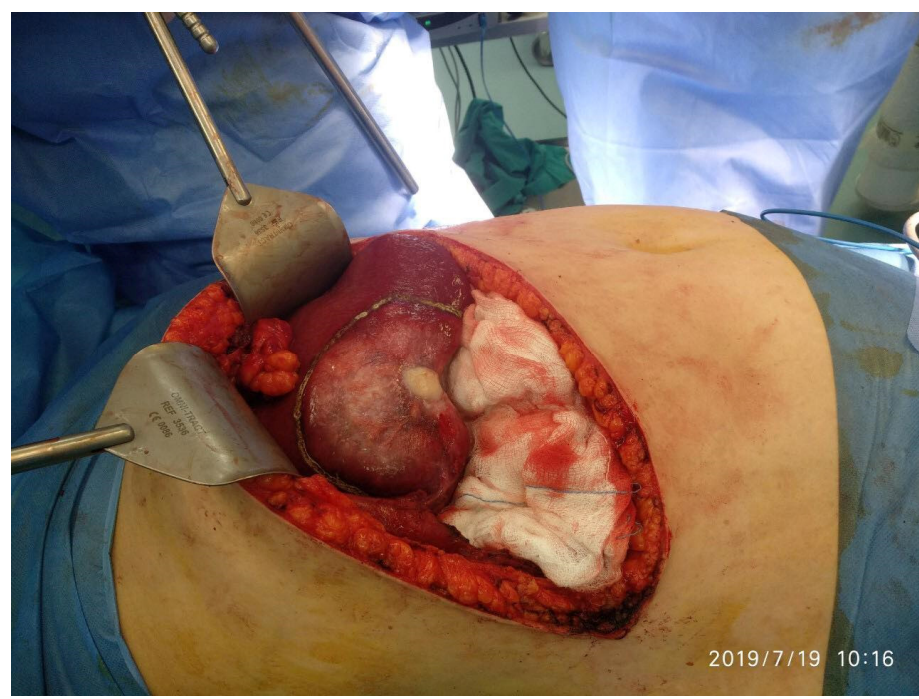

(a)

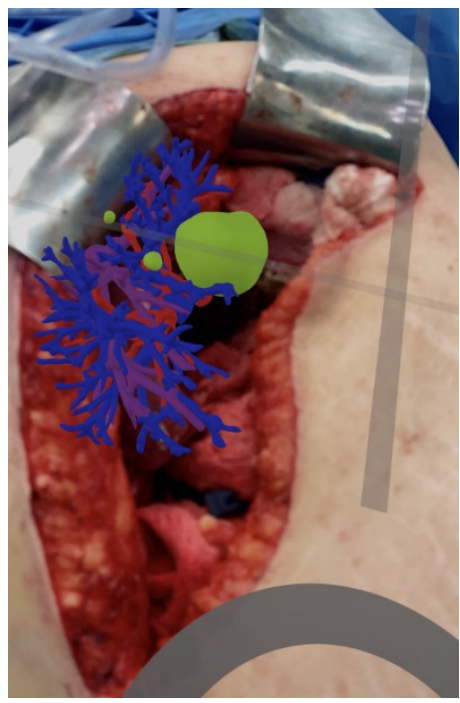

(b)

Figure 5. Surgery and augmentation. Position of the tumor as shown before the resection (a) and augmentation of the model using an iPhone camera (b). The research protocol and the study were approved by the Investigational Review Board (IRB) of the hospital where the research was conducted. Informed consent was obtained from the patient before the realization of the surgery. 


\section{Results}

In this first case, the print's final deliverable was not considered ideal, as that description would be better suited to a transparent model that contains both the liver volume, the network of blood vessels, the tumor, and the parenchyma. Therefore, initially, the vascular networks (hepatic and portal vein and arteries) should be created apart from the liver and tumor model. Using appropriate software after the production of the 3D model of the parenchyma, the extraction of the vascular network (arteries, portal, and hepatic vein) was performed from the different phases (arterial, portal, delayed) of the CT scan images. The result was the model for the next case (Figure 6), which incorporated the complete liver anatomy into a unique 3D model file (as a standard wavefront.obj digital model file).

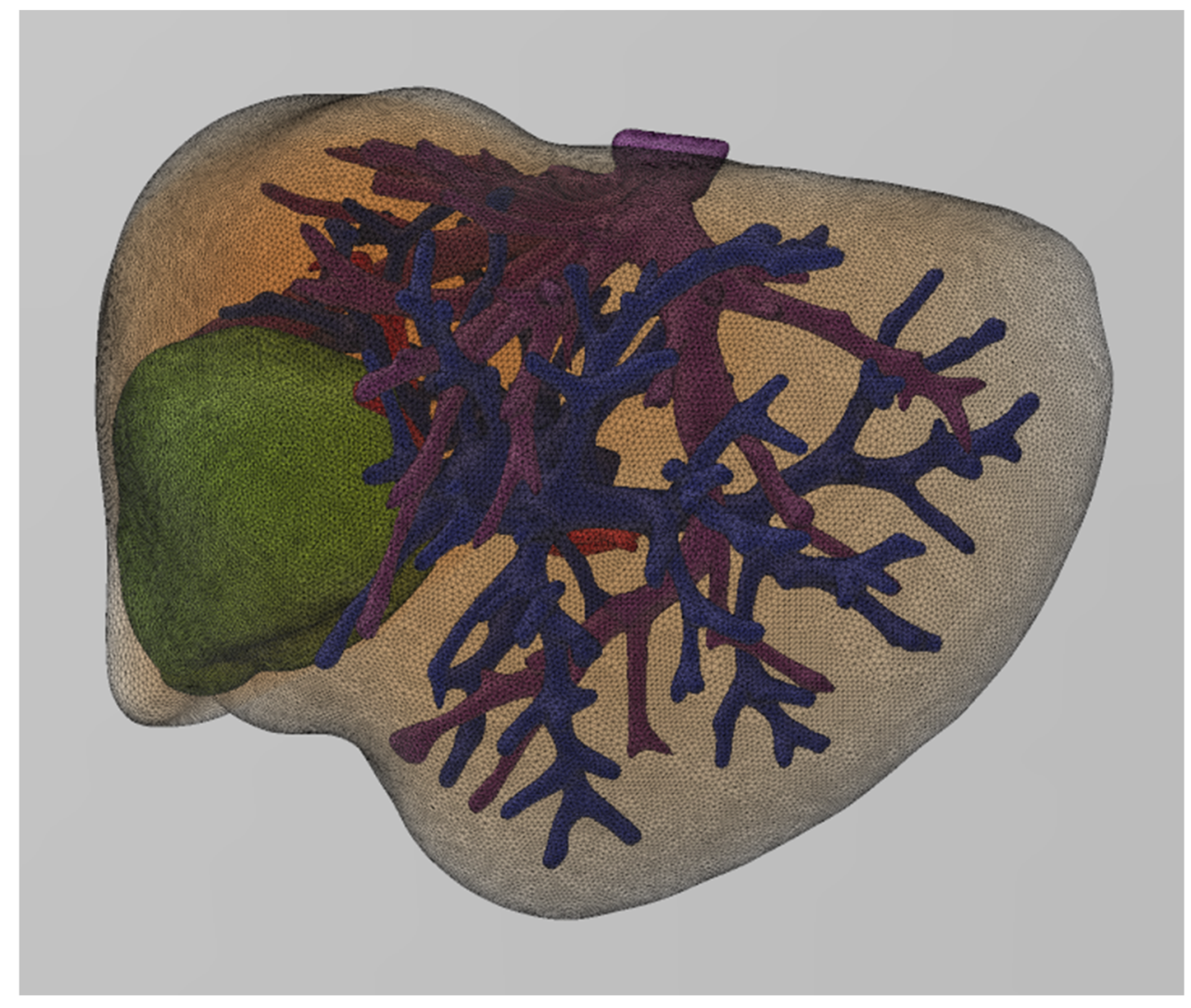

Figure 6. Patient's liver anatomy. The complete model of the patient's liver is presented. Parenchyma is presented in beige transparent color, hepatic vein in purple, aorta in red, portal vein in blue, and tumor in green.

The next challenge was to transfer to life-size (1:1 scale) a structure of a transparent object the combines the vascular networks and the liver tumor, while retaining the high precision geometric model, as it was captured from the CT scan and was subsequently digitized and modeled from our specialized software application.

A possible solution was to print a mold instead of the liver parenchyma that would integrate the vascular network and the tumor and then fill it with transparent material originally in liquid form, so as not to create gaps, and then, when solidified, to allow the study and planning-rehearsal of the operation by the surgeon.

The difficulties that arose are mainly due to the uncertainty in providing correct placement and welding of the vascular networks both with each other and in relation to the tumor, as all the models are printed separately from each other. The problem was mainly solved by the application of three-dimensional transformations between the geometric reference systems to which the individual models of the vessels refer, achieving adjustment accuracy equal to the scanning resolution of the CT scans (close to $1 \mathrm{~mm}$ ). 
An intermediate goal in the implementation of our research activities is the assessment of the results after every surgical operation. More specifically, the evaluation focuses on the comparison of the volumetric quantity resulting from the difference of the parenchyma from the liver model in relation to the tumor removed during the operation. The following (Figure 7) illustrates the resected tumor after an operation performed, as well as the corresponding 3D printed model, which was obtained originally as a digital model from the processing of the CT scans.

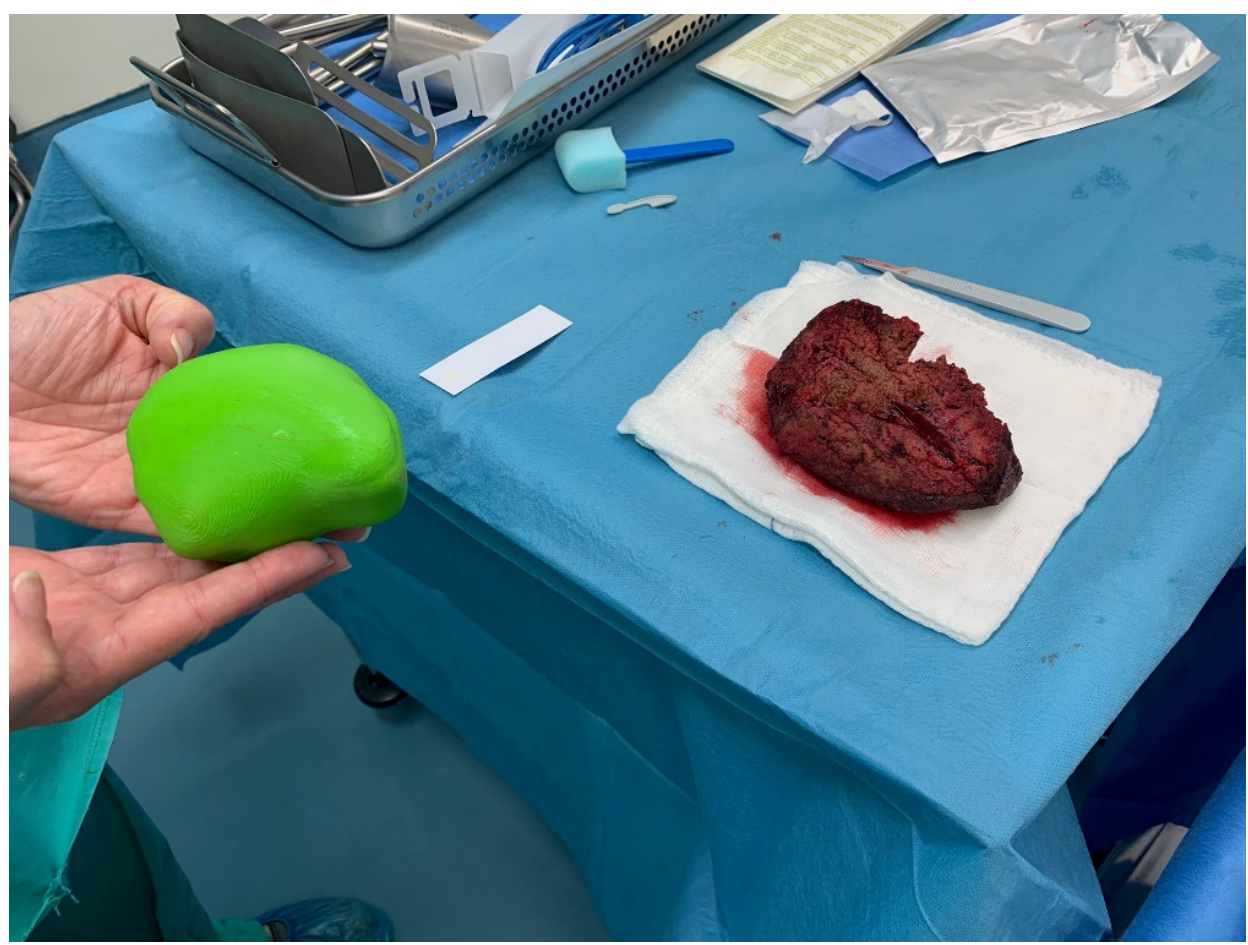

Figure 7. Resected tumor and the tumor model. A doctor is holding in his hands the 3D printed model of the tumor and is comparing it with the resected tumor lying on the surgery table. It is worthy to mention that in patients with complicated anatomy, the 3D models were more informative than even the CT.

Considering that during a surgical operation, in addition to the tumor, also margins of healthy tissue are resected for oncological reasons, the difference of $10.1 \%$ resulting from the comparative volumetric difference becomes even smaller. Therefore, the data from the volumetric comparisons encourage the application of 3D printing as an accurate method, both in terms of the location as well as the size of the lesion.

\section{Discussion}

What has been described so far is the difficult path, but one full of satisfaction and enthusiasm, that has been followed to provide an accurate illustration of the patient's disease so that the treatment would be more targeted and adapted to the specific anatomy and pathology of every single case. This approach is part of a current trend in Medicine described as "personalized medicine" or "precision medicine", and the ultimate goal is the individualized treatment of the disease in a way that would lead to fewer complications and/or side effects, greater safety and accuracy.

We chose liver and liver tumors as the subject of this research, as the complex anatomy and physiology of the liver make hepatectomy one of the most complicated surgical operations. Therefore, the assistance of advanced imaging technologies and 3D modeling methods is of critical importance both for the safety of the patient and the successful outcome of the operation. Also, with the above-mentioned mapping and 3D representation 
of the liver, it is possible to apply an even more targeted and precise resection, which means that the patient will finally retain a healthier parenchyma, without questioning the oncological success of the operation.

Another conclusion from this initial experience is the importance of this imaging method for the acquisition of more detailed preoperative information to familiarize the patients and their intimates with their pathology and disease, the operation planning, and the possible complications.

3D imaging and 3D printing of the liver and tumor on a 1:1 scale is much more convincing and informative than any information a surgeon could give. Furthermore, the 3D imaging of the liver, its anatomy, and the tumor, has enabled us to discuss and plan the surgery in advance with the rest of the surgical team, as well as to explain this strategy and possible difficulties to students and surgeons.

Finally, we believe that these are just a foretaste of what will follow, as during this project and this collaboration, there was a constant "concern" for improvement. This has led us to the next step, which is related to the conversion of the 3D printed instrument into an augmentation model that can be used during the surgery to highlight and safely deal with the dangerous points along the way of hepatectomy.

Author Contributions: Conceptualization, V.T., G.T. and C.P.; methodology, I.-A.K., C.P. and V.T.; software, I.-A.K., C.P., V.T. and M.A.; validation, A.T., G.T. and V.P.; formal analysis, V.P. and G.T.; investigation, A.T. and I.-A.K.; resources, A.T., V.P., G.T. and M.A.; data curation, V.P., A.T., I.-A.K. and M.A.; writing - original draft preparation, V.T., C.P. and G.T.; writing-review and editing, V.T., C.P. and G.T.; visualization, I.-A.K. and M.A.; supervision, V.T., V.P. and G.T.; project administration, V.T., V.P. and G.T.; funding acquisition, V.T. All authors have read and agreed to the published version of the manuscript.

Funding: Co-financed by the European Union and Greek national funds through the Operational Program Competitiveness, Entrepreneurship and Innovation, under the call RESEARCH-CREATEINNOVATE (project code: T1EDK-03599).

Institutional Review Board Statement: The study was conducted according to the guidelines of the Declaration of Helsinki, and approved by the Institutional Review Board (or Ethics Committee) of Papageorgiou General Hospital of Thessaloniki (protocol code 306/30-11-2018).

Informed Consent Statement: Informed consent was obtained from all subjects involved in the study.

Data Availability Statement: All data used in the current study are stored in the "Papageorgiou General Hospital of Thessaloniki" picture archiving and communication system (PACS).

Conflicts of Interest: The authors declare no conflict of interest.

\section{References}

1. Haryńska, A.; Janik, H.; Sienkiewicz, M.; Mikolaszek, B.; Kucińska-Lipka, J. PLA-Potato Thermoplastic Starch Filament as a Sustainable Alternative to the Conventional PLA Filament: Processing, Characterization, and FFF 3D Printing. ACS Sustain. Chem. Eng. 2021, 9, 6923-6938. [CrossRef]

2. Oliveira, D.A.; Feitosa, R.Q.; Correia, M.M. Segmentation of liver, its vessels and lesions from CT images for surgical planning. Biomed. Eng. Online 2011, 10, 30. [CrossRef]

3. Yang, X.; Do Yang, J.; Hwang, H.P.; Yu, H.C.; Ahn, S.; Kim, B.W.; You, H. Segmentation of liver and vessels from CT images and classification of liver segments for preoperative liver surgical planning in living donor liver transplantation. Comput. Methods Programs Biomed. 2018, 158, 41-52. [CrossRef] [PubMed]

4. Ngo, T.D.; Kashani, A.; Imbalzano, G.; Nguyen, K.T.; Hui, D. Additive manufacturing (3D printing): A review of materials, methods, applications and challenges. Compos. B Eng. 2018, 143, 172-196. [CrossRef]

5. Tack, P.; Victor, J.; Gemmel, P.; Annemans, L. 3D-printing techniques in a medical setting: A systematic literature review. Biomed. Eng. Online 2016, 15, 115. [CrossRef] [PubMed]

6. Hull, C.W. Apparatus for Production of Three-Dimensional Objects by Stereolithography. U.S. Patent US4575330, 11 March 1986. pp. 1-16.

7. Tsoulfas, G.; Bangeas, P.I.; Suri, J. 3D Printing: Applications in Medicine and Surgery, 1st ed.; Elsevier: Amsterdam, The Netherlands, 2019.

8. Giannopoulos, A.A.; Steigner, M.L.; George, E.; Barile, M.; Hunsaker, A.R.; Rybicki, F.J.; Mitsouras, D. Cardiothoracic Applications of 3-dimensional Printing. J. Thorac. Imaging 2016, 31, 253-272. [CrossRef] [PubMed] 
9. Chae, M.P.; Rozen, W.M.; McMenamin, P.G.; Findlay, M.W.; Spychal, R.T.; Hunter-Smith, D.J. Emerging Applications of Bedside 3D Printing in Plastic Surgery. Front. Surg. 2015, 2, 25. [CrossRef]

10. Malik, H.H.; Darwood, A.R.J.; Shaunak, S.; Kulatilake, P.; El-Hilly, A.A.; Mulki, O.; Baskaradas, A. Three-dimensional printing in surgery: A review of current surgical applications. J. Surg. Res. 2015, 199, 512-522. [CrossRef] [PubMed]

11. Tsioukas, V.; Karolos, I.A.; Tsoulfas, G.; Suri, J.S.; Pikridas, C. The long and winding road from CT and MRI images to 3D models. In 3D Printing: Applications in Medicine and Surgery; Elsevier: Amsterdam, The Netherlands, 2020; pp. 7-20.

12. A Matter of Grayscale: Understanding Dicom Windows. Available online: https://towardsdatascience.com/a-matter-ofgrayscale-understanding-dicom-windows-1b44344d92bd (accessed on 18 August 2021).

13. 2.5D (Visual Perception). Available online: https://en.wikipedia.org/wiki/2.5D_(visual_perception) (accessed on 18 August 2021).

14. Singh, A.V.; Romeo, A.; Scott, K.; Wagener, S.; Leibrock, L.; Laux, P.; Luch, A.; Kerkar, P.; Balakrishnan, S.; Dakua, S.P.; et al. Emerging Technologies for In Vitro Inhalation Toxicology. Adv. Healthc. Mater. 2021, 2100633. [CrossRef] [PubMed] 